\title{
A Novel Signal Detection Algorithm for a Wide Band Detection-Finding System in the HF Band
}

\author{
Qin Shi, Jin-yu Xiong, Hua-feng Peng \\ Science and Technology on Blind Signal Processing \\ Laboratory \\ Chengdu, China \\ ahsqff@gmail.com
}

\author{
Lai-yuan Luo \\ Southwest Electronics and Telecommunication \\ Technology Research Institute \\ Chengdu, China
}

\begin{abstract}
This article describes a novel multi-dimension combination signal detection algorithm based on the spacefrequency-energy domain for the raw data derived from high frequency (HF) wide band direction-finding (DF) systems. According to the HF wide band DF data, the time-frequencypower spectrum and its corresponding azimuth information are first transformed into azimuth-frequency-energy spectrum of DF data along the time axis; then the noise characteristics of azimuth-frequency-energy spectrum are analyzed and the detection threshold is achieved by the Neyman-Pearson (N-P) criterion at one certain false alarm probability $\left(P_{F A}\right)$; finally the signals are detected by comparing the energy of every azimuth-frequency point with the detection threshold. Through simulation and raw data validation, this algorithm can detect the weak signals of HF wide band DF data.
\end{abstract}

Keywords-projection transform; noise characteristics; N-P criterion; signal detection; $\mathrm{HF}$ wide band $\mathrm{DF}$;

\section{INTRODUCTION}

When HF wide band direction-finding (DF) systems measure the azimuth of the coming waves[1], the result of DF has many outliers and consists of the azimuths of signal , noise and interference because of the effects of the frequency selective HF channels, the non-stationary HF noise , the non-stationary signal and so on. It is difficult to obtain the signal azimuth and achieve the accurate value of the automatic wideband intersecting location which is based on multi-station DF data matching. In order to obtain the accurate azimuth of signal, it is important to detect the signal first.

Sheng-en Luo [2] and Ming Ye [3] et al have carried out related studies of signal detection and sorting based on DF wide band received signals. Franz Quint [4][5] and Frank Raps[6] et al have carried out related studies of signal detection and emitter classification ,but their methods are based on the template matching of image processing. There are some limitations to their approaches and has been little research in the literature about the signal detection based on HF wide band DF data.

\section{BASIC PRINCIPLE OF ALGORITHM}

The time-frequency-azimuth spectrum and timefrequency -power spectrum based on the HF wideband DF data which consists of time, frequency, azimuth, elevation , power, signal quality and other information are shown in Fig 1and Fig 2 respectively.

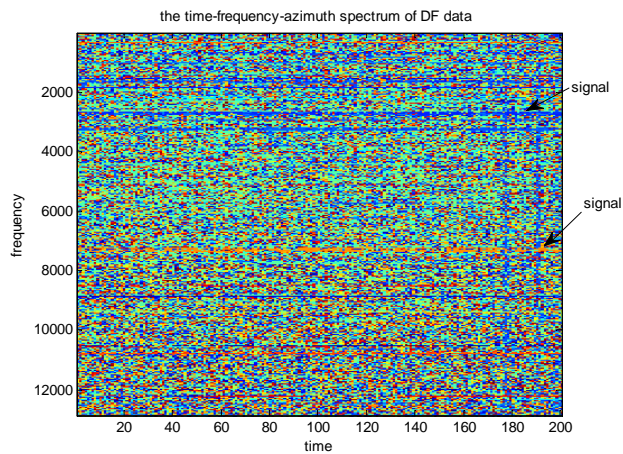

Figure 1 DF data time-frequency-azimuth spectrum

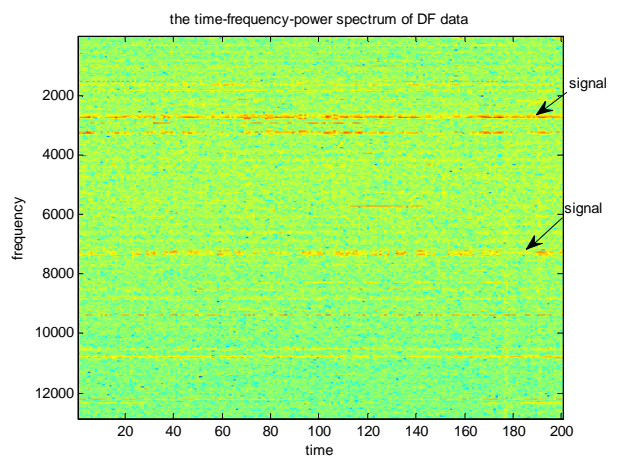

Figure 2 DF data time-frequency-power spectrum

The color of the two figures denotes the azimuth and power value respectively. As illustrated in Fig 1 and Fig 2, there are signals as well as noise. It affects the detection of the signals.

In general, the emitters with different positions have different azimuths in HF wide band DF systems [4]. The azimuth value of signal affected by noise is a Gaussian process, but the noise azimuth value follows the random distribution. Moreover long duration integration of the interesting signal energy is a key to develop an effective detector under severe noise background in many practical applications [7][8].

So the wide band DF data time-frequency-power spectrum and its corresponding azimuth information are transformed into data azimuth-frequency-energy spectrum along the time axis; because of the different characteristics of signal and noise, signal is focused in the azimuth-frequency 
domain. But noise is distributed in the azimuth-frequency domain uniformly, Then the statistic characteristics of noise in the azimuth-frequency-energy spectrum is analyzed. Finally the signals are detected by the N-P criterion in the azimuth-frequency-energy domain. The specific process is shown in Fig 3.

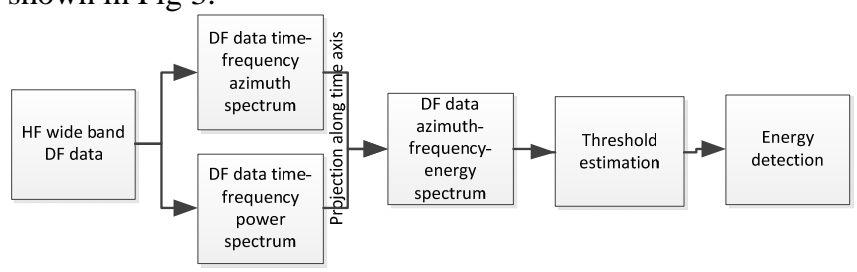

Figure 3 algorithm flow chart

\section{A. Projection Algorithm}

Time series multi-frame projection in image processing is a process which transforms image sequences from threedimension spatio-temporal domain to two-dimensional space domain to obtain singularity combination image by lossy compression [9]. Common projection algorithm consists of maximum projection algorithm, summation projection algorithm and so on

- $\quad$ Maximum value projection algorithm

$$
z(x, y) \approx \max [r(x, y, t), t=1, \cdots, N]
$$

Where $r(x, y, t)$ denotes the pixel value of one frame, $z(x, y)$ denotes the pixel value after projection algorithm. As illustrated by(1), the pixel value after the maximum projection algorithm is the max value of the related position pixel of image sequence.

- Summation projection algorithm

$$
z(x, y) \approx \sum_{t=1}^{N} r(x, y, t)
$$

Where $r(x, y, t)$ denotes the pixel value of one frame, $z(x, y)$ denotes the pixel value after summation projection algorithm. As illustrated by(2), the pixel value after summation projection algorithm is the summation of the related position pixel of image sequence.

Considering the method of summation projection algorithm in image processing, the power information of HF wide band DF data is added into azimuth-frequency domain by compressing time dimension, which benefits the signal detection .Specific process is shown below:

$$
\begin{gathered}
\theta=H(t, f) \\
E_{N}(\theta, f)=\sum_{t=1}^{N} P(\theta, f, t)
\end{gathered}
$$

Where $t=1, \cdots, N, N$ denotes the projection time length, $H(t, f)$ denotes the azimuth of frequency $f$ at time $t, \theta$ denotes the azimuth of frequency $f$ at time $t$, $P(\theta, f, t)$ denotes the power value of frequency $f$ and azimuth $\theta$ at time $t . E_{N}(\theta, f)$ denotes the energy value of frequency $f$ and azimuth $\theta$ after projection transform. As illustrated by all the equations above, it can be seen that the projection algorithm adds the power values of all frequencies and azimuths in every time into the corresponding frequency and azimuth points in azimuth-frequency domain. So the azimuth-frequency-energy spectrum is achieved, which is shown below.

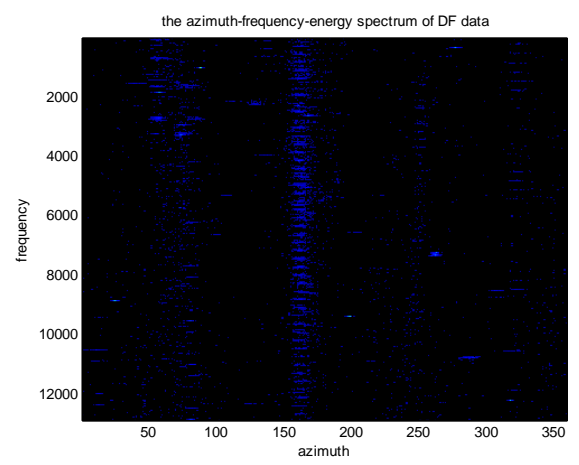

Figure 4 the azimuth-frequency-energy spectrum of DF data

According to the related knowledge in mathematical statistics [10], the summation projection by compressing time dimension realizes the cumulation of energy in DF data. Because the different characteristics of signal and noise, signal is focused in the azimuth-frequency domain. But noise is distributed in the azimuth-frequency domain uniformly.

\section{B. Noise Characteristics Analysis}

Signal detection mainly consists of threshold estimation, energy detection and so on. The function of threshold estimation determines the noise floor of azimuth-frequencyenergy domain and calculates the detection threshold according to the false alarm probability $\left(P_{F A}\right)$ Energy detection compares the energy of azimuth-frequency points with the detection threshold in order to find the points which have signal [11].

Supposing that the HF noise is stationary in the frequency range of $2 \mathrm{MHz}$ in short time, the wide band received signals of $\mathrm{HF}$ DF systems are digitized and converted into blocks of multichannel data with FFT processing in some time intervals. The window function is rectangle. Namely, the discrete received signal $r(n)$ is converted to time-frequency domain by STFT :

$$
\begin{gathered}
r(n)=s(n)+n(n) \\
\operatorname{TFR}_{n}(k)=\sum_{k=0}^{N-1} r(m) w(n-m) e^{-2 \pi k m / N}
\end{gathered}
$$

Where $s(n)$ denotes the discrete signal sequence, $n(n)$ denotes the noise sequence, $N$ is the length of $\operatorname{STFT}, w(n)$ is a window function.

Considering the wide band received signal $r(k)$,if $n(k)$ is a white Gaussian process of zero mean and variance $\delta^{2}$ and the window function $w(k)$ is a boxcar window, the 
time-frequency point power value without signals(the power value is $y$ ) follows central $\chi^{2}$ with two degrees of freedom and proportionality parameter $\delta^{2} / 2[12]-[14]$

$$
f(y)=\frac{\delta^{2}}{2} F(y \mid D)
$$

Where the degrees of freedom $D$ equals to 2 , $F(y \mid D)$ denotes the probability density function (PDF) of central $\chi^{2}$ with $D$ degrees of freedom.

The expected value and variance of y are derived from(7):

$$
E(y)=\delta^{2} \quad D(y)=\delta^{4}
$$

The problem of signal detection based on HF wide band DF data can be converted to the problem of hypothesis test.

It can be concluded that if the projection length of $K$ is large sufficiently, the summation of $K$ identically distributed (IID ) $\chi^{2}$ random variables follows the Gaussian distribution by the central limit theorem (CLT) [10].

The PDF of noise energy $x$ follows the Gaussian distribution:

$$
f_{H_{0}}(x)=\frac{1}{\sqrt{2 \pi} \delta_{n}} e^{-\frac{\left(x-u_{n}\right)^{2}}{2 \delta_{n}^{2}}}
$$

Where $u_{n}$ and $\delta_{n}^{2}$ denote the expected value and variance of $x$ respectively.

$$
u_{n}=E(x)=K \delta^{2}, \quad \delta_{n}^{2}=D(x)=K \delta^{4}
$$

Supposing that the power of signal is constant, the PDF of the energy $x$ of the azimuth-frequency point with signal under $H_{1}$ hypothesis is showed as following [12]:

$$
f_{H_{1}}(x)=\frac{1}{\sqrt{2 \pi} \delta_{n}} e^{-\frac{\left(x-\left(u_{n}+u_{s}\right)\right)^{2}}{2 \delta_{n}^{2}}}
$$

Where $u_{s}$ denotes the signal expected value after the projection transform. $K$ denotes the cumulative time length $u_{s}=K P_{s} . P_{s}$ denotes the power of signal. Because the power of signal is constant, the variance of signal is zero.

By using the N-P detection criterion in practical project frequently, the detection statistics can be derived from the likelihood ratio test.

The decision rule under the N-P detection criterion is shown as following [11]:

$$
\frac{f_{H_{1}}(x)}{f_{H_{0}}(x)} \stackrel{H_{1}}{>} \text { th }
$$

And $f_{H_{1}}(x) / f_{H_{0}}(x)$ can be expressed as:

$$
\frac{f_{H_{1}}(x)}{f_{H_{0}}(x)}=\frac{\frac{1}{\sqrt{2 \pi} \delta_{n}} e^{-\frac{\left(x-u_{n}\right)^{2}}{2 \delta_{n}^{2}}}}{\frac{1}{\sqrt{2 \pi} \delta_{n}} e^{-\frac{\left(x-u_{n}-u_{s}\right)^{2}}{2 \delta_{n}^{2}}}}=e^{\frac{2 u_{s} x-2 u_{n} u_{s}-u_{s}^{2}}{2 \delta_{n}^{2}}}
$$

Substituting (13) into(12) , (12) can be simply written as:

$$
x<\frac{2 \delta_{n}^{2} \ln t h+2 u_{n} u_{s}+u_{s}^{2}}{2 u_{s}}
$$

Where $t h^{\prime}$ is defined as: $t h^{\prime}=\frac{2 \delta_{n}^{2} \ln t h+2 u_{n} u_{s}+u_{s}^{2}}{2 u_{s}}$

And the decision rule (14) can be simply expressed as:

$$
x_{H_{0}}^{>} t h^{\prime}
$$

The detection threshold $t h^{\prime}$ is solved according to $P_{F A}$ and the PDF of noise $f_{H_{0}}(x)$.The specific process is shown as following [15]:

$$
\begin{aligned}
P_{F A} & =\int_{t h^{\prime}}^{+\infty} f_{H_{0}}(x) d x=\int_{t h^{\prime}}^{+\infty} \frac{1}{\sqrt{2 \pi} \delta_{n}} e^{-\frac{\left(x-u_{n}\right)^{2}}{2 \delta_{n}^{2}}} d x \\
& =1-\Phi\left(\frac{t h^{\prime}-u_{n}}{\delta_{n}}\right)
\end{aligned}
$$

The detection threshold $t h^{\prime}$ is shown as following [16]:

$$
t h^{\prime}=\delta_{n} \Phi^{-1}\left(1-P_{F A}\right)+u_{n}
$$

Where $\Phi(x)$ is defined as $\Phi(x)=\int_{-\infty}^{x} \frac{1}{\sqrt{2 \pi}} e^{-\frac{t^{2}}{2}} d t$ and $\Phi^{-1}(x)$ is the inverse function of $\Phi(x)$.

From all the equations above, if the energy $x$ of azimuth-frequency point is higher than the threshold $t h^{\prime}$, we think that the point is a signal point, otherwise the point is not a signal point. So the detection probability $\left(P_{D}\right)$ is expressed as following [17]:

$$
\begin{aligned}
P_{D} & =\int_{t h^{\prime}}^{+\infty} f_{H_{1}}(x) d x=\int_{t h^{\prime}}^{+\infty} \frac{1}{\sqrt{2 \pi} \delta_{n}} e^{-\frac{\left(x-u_{n}-u_{s}\right)^{2}}{2 \delta_{n}^{2}}} d x \\
& =1-\Phi\left(\frac{t h^{\prime}-u_{n}-u_{s}}{\delta_{n}}\right)
\end{aligned}
$$

Substituting (17) into (18), (18)can be simply expressed as following:

$$
\left.P_{D}=1-\Phi\left(\Phi^{-1}\left(1-P_{F A}\right)-\frac{u_{s}}{\delta_{n}}\right)\right)
$$


As illustrated $\operatorname{in}(20), P_{D}$ is increased versus $u_{s} / \delta_{n}$ increased. And

$$
\begin{gathered}
\frac{u_{s}}{\delta_{n}}=\frac{K P_{s}}{\sqrt{K \delta^{4}}}=\sqrt{K} \frac{P_{s}}{\delta^{2}}=\sqrt{K} S N R \\
S N R=\frac{P_{s}}{\delta^{2}}
\end{gathered}
$$

It is a fact that if the cumulative length $K$ or the signalnoise-ratio (SNR) is increased, $P_{D}$ is increased.

\section{Analysis of Detection Performance}

From the analysis above, it is a fact that $P_{D}$ is increased versus the equivalent energy-to-noise ratio $u_{s} / \delta_{n}$ and $u_{s} / \delta_{n}=\sqrt{K} S N R$.So if the projection time length $K$ or

$S N R$ are increased, the detection performance is increased.

The detection performance versus equivalent energy-toratio is shown in Fig 5.

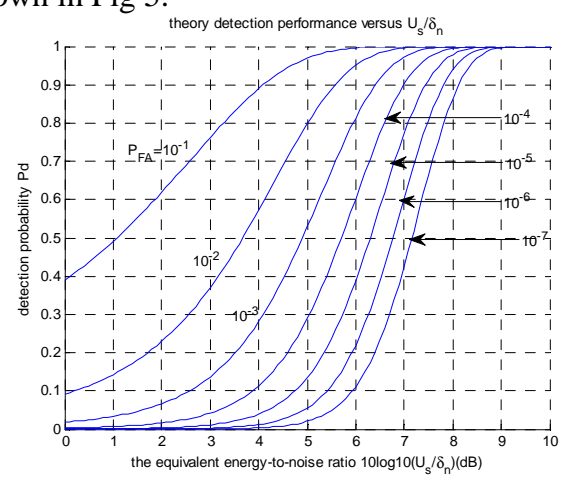

Figure 5 the theory detection performance versus the equivalent energy-tonoise ratio $u_{s} / \delta_{n}$

As illustrated in Fig 5, when $P_{F A}$ is constant, $P_{D}$ is increased versus the equivalent energy-to-noise ratio $u_{s} / \delta_{n}$ increased.

The detection performance versus $S N R$ is shown in Fig 6 and Fig 7 when the projection time length is changed.

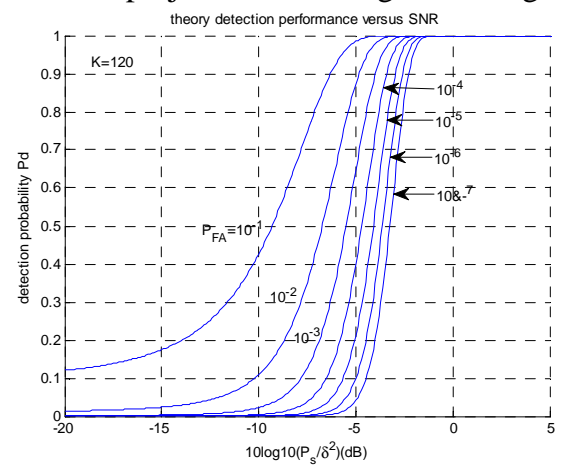

Figure 6 the theory detection performance versus $S N R$ when the projection time length equals to 120

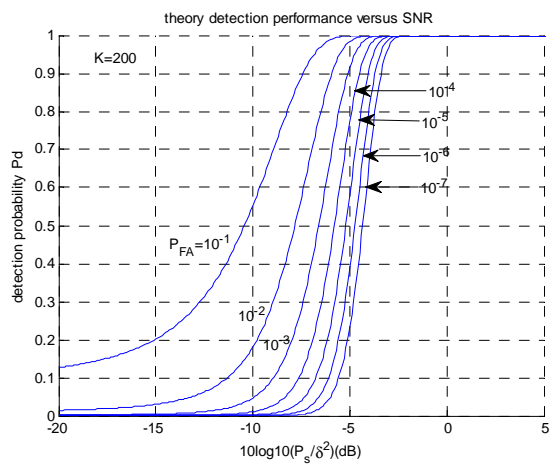

Figure 7 the theory detection performance versus $S N R$ when the projection time length equals to 200

As illustrated in the two figures above, when the projection time length $K$ and $P_{F A}$ are constant, $P_{D}$ is increased versus $S N R$ increased. And When $P_{F A}$ is constant, if the value of the projection time length $K$ is larger, it needs less SNR in order to achieve the same $P_{D}$ value.

\section{SIMULATION AND EXPERIMENT VALIDATION}

\section{A. Simulation Validation}

The detection performance changing with the equivalent energy-to-noise ratio $u_{s} / \delta_{n}, S N R$ and projection time length $K$ is analyzed below. In the simulation, the projection time length $K$ is set to 120 and 200.The $P_{F A}$ value is $10^{-1}, 10^{-2}, \ldots, 10^{-7}$. The length of frequency region is 360 and the length of azimuth region is 20.the noise power value follows the central $\chi^{2}$ with two degrees of freedom in the time-frequency domain. The point of azimuth-frequency domain is served as the object in order to analyze the performance versus the equivalent energy-tonoise ratio $u_{s} / \delta_{n}$. The result is shown in Fig 8.

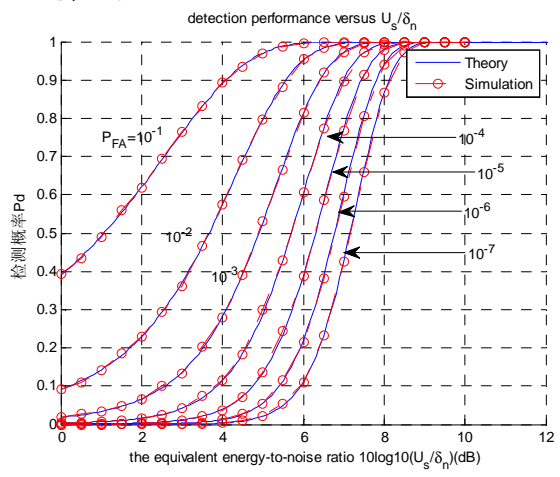

Figure 8 the comparison with the theory and simulation detection performance versus the equivalent energy-to-noise ratio $u_{s} / \delta_{n}$

The detection performance versus the $S N R$ with different projection time length $K$ is shown in Fig 9 and Fig 10. 


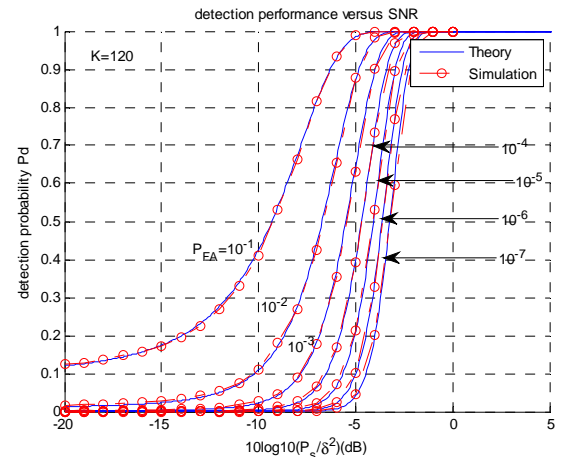

Figure 9 the comparison with the theory and simulation detection performance versus SNR when the projection time length equals to 120

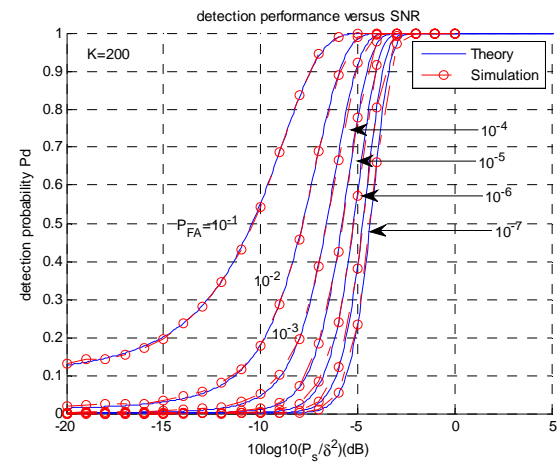

Figure 10 the comparison with the theory and simulation detection performance versus SNR when the projection time length equals to 200

It can be concluded from the simulation result and theory result that the algorithm described in this paper can detect the weak signals of HF wide band DF data by increasing the projection time length.

\section{B. Experiment Validation}

The raw data is received from the HF wide band DF systems. The center frequency is $7 \mathrm{MHz}$ and the bandwidth is $2 \mathrm{MHz}$. The sample frequency is $2.56 \mathrm{MHz}$. The time and frequency intervals are respectively $26 \mathrm{~ms}$ and $125 \mathrm{~Hz}$. The time length of the DF data is 200.The time-frequency azimuth spectrum and time-frequency power are shown in Fig 11 and Fig 12.

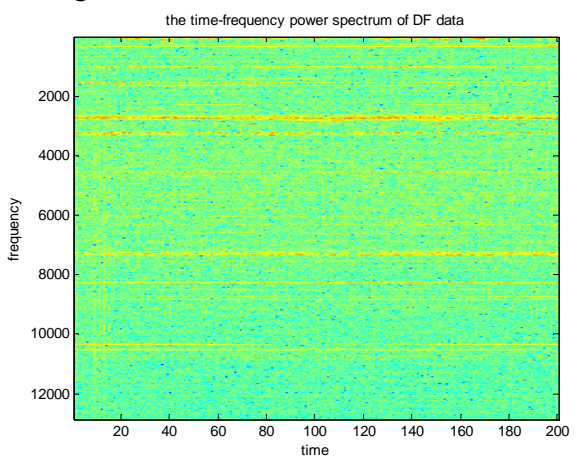

Figure 11 the time-frequency power spectrum of raw DF data

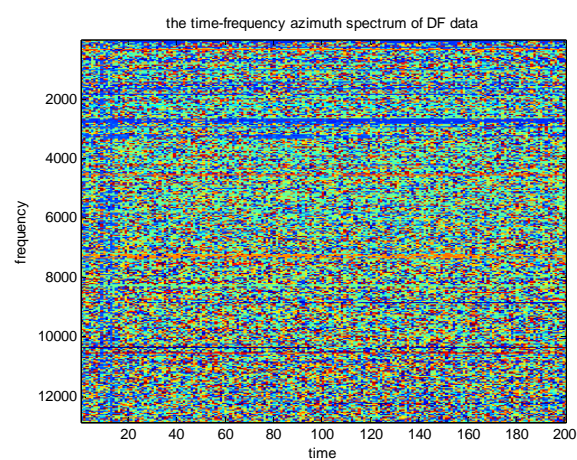

Figure 12 the time-frequency azimuth spectrum of raw DF data The azimuth-frequency-energy spectrum after projection along time axis is presented in Fig 13.

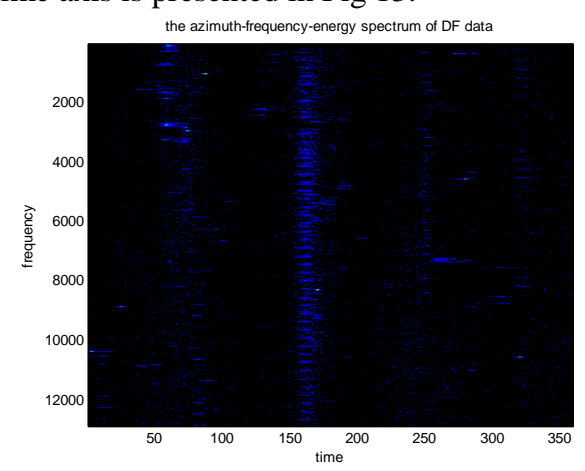

Figure 13 the azimuth-frequency-energy spectrum of DF data

The histogram of the azimuth-frequency-energy spectrum is presented in Fig 14.The statistic range is from 0 to 2000 by the prior knowledge of noise energy value range.

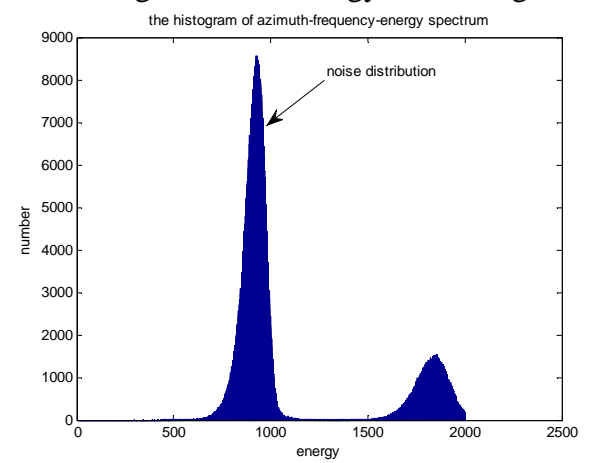

Figure 14the histogram of the azimuth-frequency-energy spectrum

The noise PDF of the azimuth-frequency-energy spectrum is fitted by the Gaussian distribution. The detection threshold is derived from (17) when $P_{F A}$ is $10^{-3}$.The result of the signal detection by comparing the energy of every azimuth-frequency point with the detection threshold is shown in Fig 15. 


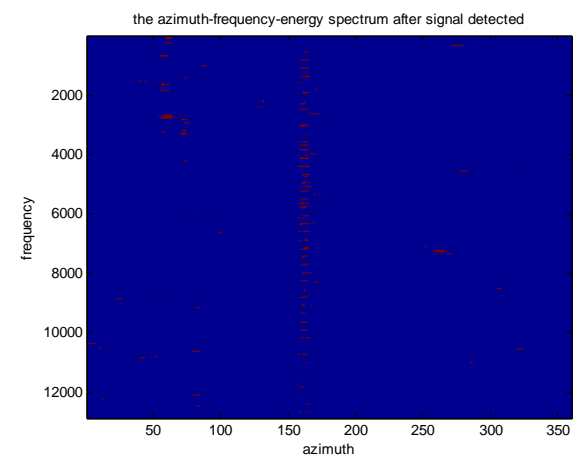

Figure 15 the azimuth-frequency-energy spectrum after signal detected

As illustrated in Fig 15, the algorithm proposed by this paper can achieve the detection of weak signal based on the HF wide band DF data.

\section{CONCLUSION}

A multi-dimension combination signal detection algorithm based on the space-frequency-energy domain is proposed in this paper aiming at the target of detecting the signal of the HF wide band DF data. Validated by the simulation and experiment, the algorithm can detect the weak signal of HF wide band DF data by increasing the projection time length. It can support the accurate acquisition of every signal of DF data and the automatic wideband intersecting location which is based on multi-station DF data matching. Further work concerning the signal segmentation is in progress.

\section{ACKNOWLEDGMENT}

The authors are grateful to the reviewers for their helpful suggestions.

\section{REFERENCES}

[1] Qi-qi Shen,De-sheng Zhu,’High frequency communication,’Xi'an: Xidian University Press,1989.

[2] Sheng-en Luo,"Research on the key techniques for wideband processing based on HF signal sortation," Chengdu: Southwest Electronics and Telecommunication Technology Research Institute
[3] Ming Ye, "The research on classification of HF specific signals using visual features of wideband spectrogram," Chengdu: Southwest Electronics and Telecommunication Technology Research Institute.

[4] Franz Quint, Juergen Reichert, Holger Roos. Emitter detection and tracking algorithm for a wide band multichannel direction-finding system in the HF-Band. IEEE, pp. 139-142, 1999.

[5] Peter Ahlemann, Martin Kueckenwaitz , Franz Quint, et al. An automated monitoring system for the HF band. IEEE, pp.139$142,2001$.

[6] Frank Raps, Kuno Kollmann, Hans Christoph Zeidler, HF-BAND emitter detection and segmentation based on image processing. IEEE,pp. 428-431, 2001.

[7] Hai-yan Shang,Peng-lang Shui,Shou-hong Zhang, et al, ”Energy integration detection via time-frequency distributionand morphological filtering," Journal of Electronics \& Information Technology, Vol.29, No.2,pp. 1416-1420.

[8] Hai-hua Zhu, Ze-ming Wang,” A new algorithm of signal detection based on time-frequency distribution," Guidance and Fuze, Vol.29,No.1,pp.38-43,Mar 2008.

[9] Ming-yu Cong,Wen-jia He,Wen-zhuo Bao, et al, "Suppression of cluttered cloud image background by time series multi-frame projectio,” Optics and Precision Engineering,Vol.20,No.4,Apr 2012.

[10] Zhou Sheng, Shi-qian Xie,Chen-yi Pan,"Probability theory and mathematical statistics,” Beijing:Higher Education Press,2008.

[11] Steven M. Kay,"Fundamentals of statistical signal processingestimation and detection theory,"Beijing:Publishing House of Electronics Industry,2011.

[12] Paul. E . Johnson, David G. Long. The probability density of spectral estimates based on modified periodogram averages. IEEE Trans. Signal Processing.Vol.47, pp.1255-1261,1999.

[13] L. H. Koopmax. The spectral analysis of time series. New York: Academic, 1964

[14] Cyril Hory, Nadine Martin, Alain Chehikian.,’Spectrogram segmentation by means of statistical features for non-stationary signal interpretation,” IEEE Trans . Signal Processing, Vol. 50 , NO. 12, pp. 2915-2925, 2002.

[15] You He,Jian Guan,Xiang-wei Meng,’Radar target detection and CFAR processing,’Beijing:TsingHua University Press,2011.

[16] Khiem V Cai, Vu Phan, Roger J O’Connor. Energy detector performance in a noise fluctuating channel. IEEE, pp. 85-89,1989.

[17] Fadel Digham, Mohamed-Slim Alouini , Marvin K. Simon. On the energy detection of unknown signals over fading channels. IEEE, pp. $3575-3579,2003$ 